\title{
Intuitionistic Fuzzy Soft Groups Induced by (t, s)-norm
}

\author{
Naveed Yaqoob', Muhammad Akram²* and Muhammad Aslam ${ }^{3}$ \\ 1,3Department of Mathematics, Quaid-i-Azam University, Islamabad, Pakistan; \\ nayaqoob@ymail.com,draslamqau@yahoo.com \\ 2Department of Mathematics, University of Gujrat, Gujrat, Pakistan; makram_69@yahoo.com
}

\begin{abstract}
This article concerns the relationship between intuitionistic fuzzy soft sets and groups. In this paper, the notion of intuitionistic fuzzy soft groups is introduced and $(\lambda, \theta)$-level set, union and intersection of them are studied. Furthermore, definition of direct product of intuitionistic fuzzy soft group under soft function is defined.
\end{abstract}

Keywords: Soft sets; Intuitionistic fuzzy soft sets; Intuitionistic fuzzy soft groups.

2010 AMS Classification: 20N25, 03E72, 03F55.

\section{Introduction}

The concept of soft set was given by Molodtsov [22], which is a completely new approach for modeling and uncertainty. A soft set is a parameterized family of subsets of the universal set. We can say that soft sets are neighborhood systems, and that they are a special case of context-dependent fuzzy sets. In soft set theory the problem of setting the membership function, among other related problems, simply does not arise. This makes the theory very convenient and easy to apply in practice. Aktas and Cagman [7] introduced the basic properties of soft sets. Jun [17] applied soft sets to the theory of $\mathrm{BCK} / \mathrm{BCI}$-algebras, and introduced the concept of soft BCK/BCI-algebras. Abdullah et al. [1-4] defined fuzzy sets in different algebraic structures. Yaqoob et al. [25] applied soft set theory to $\Gamma$-hyperideals in left almost $\Gamma$-semihypergroups, also see [26-28]. Feng et al. [15] defined soft semirings and several related notions to establish a connection between soft sets and semirings. Ali and Shabir [8] defined soft ideals and generalized fuzzy ideals in semigroups. Maji et al. [21] presented the definition of fuzzy soft set. Some corrections were given by Ali et al. [9]. Aygunoglu and Aygun [13] introduced the concept of fuzzy soft group and in the meantime, discuss some properties

\footnotetext{
${ }^{*}$ Corresponding author:
}

Muhammad Akram (makram_69@yahoo.com) and structural characteristics of fuzzy soft group. Maji et al. $[19,20]$ introduced the notion of the intuitionistic fuzzy soft set theory which is based on a combination of the intuitionistic fuzzy set $[10,11]$ and soft set models.

Kim et al. [18] introduced the concept of intuitionistic $(T, S)$ normed fuzzy ideals of $\Gamma$-rings. Akram and Dar [5] introduced the idea of fuzzy left h-ideal in hemirings with respect to an s-norm. Zhan [29], studied the fuzzy left h-ideals in hemirings with t-norms. There are several authors who applied the theory of intuitionistic $(S, T)$-fuzzy sets to different algebraic structures for instance, Akram [6], Aygunoglu et al. [12], Borumand Saeid and Rezaei [14], Hedayati [16] and Shum et al. [24].

In this paper, the notion of intuitionistic fuzzy soft groups is introduced and $(\lambda, \theta)$-level set, union and intersection of them are studied.

\section{Review of Literature}

Molodtsov defined the notion of a soft set as follows. Let $U$ be an initial universe and $E$ be the set of parameters. Usually, parameters are attributes, characteristics or properties of an object. Let $P(U)$ denote the power set of $U$ and $A$ is a subset of $E$. 
DEFINITION 2.1 [22] A pair (F, A) is called a soft set over $U$, where $F$ is a mapping given by $F: A \rightarrow P(U)$. In other words a soft set over $U$ is a parameterized family of subsets of $U$.

DEFINITION 2.2 [22] Two soft sets $(F, A)$ and $(G, B)$ over a common universe $U$ are said to be soft equal if $(F, A)$ is a soft subset of $(G, B)$ and $(G, B)$ is a soft subset of $(F, A)$.

Definition 2.3 An intuitionistic fuzzy set $A$ in $X$ is an object having the form

$$
A=\left\{\left(x, \mu_{A}(x), \gamma_{A}(x)\right): x \in X\right\},
$$

where the function $\mu_{A}: X \rightarrow[0,1]$ and $\gamma_{A}: X \rightarrow[0,1]$ denote the degree of membership and the degree of non-membership of each element $x \in X$, and $0 \leq \mu_{A}(x)+\gamma_{A}(x) \leq 1$ for all $x \in X$. For the sake of simplicity, we will use the symbol $A=\left(\mu_{A}, \gamma_{A}\right)$ for the intuitionistic fuzzy set $A=\left\{\left(x, \mu_{A}(x), \gamma_{A}(x)\right): x \in X\right\}$. We define $0(x)=0$ and $1(x)=1$ for all $x \in X$.

Definition 2.4 [10] Let $A=\left(\mu_{A}, \gamma_{A}\right)$ and $B=\left(\mu_{B}, \gamma_{B}\right)$ be intuitionistic fuzzy sets in a set $S$. Then

(1) $A \subseteq B$ if and only if $\mu_{A} \leq \mu_{B}$ and $\gamma_{A} \geq \gamma_{B}$,

(2) $A^{c}=\left(\gamma_{A}, \mu_{A}\right)$,

(3) $A \cap B=\left(\mu_{A} \wedge \mu_{B}, \gamma_{A} \vee \gamma_{B}\right)$,

(4) $A \cup B=\left(\mu_{A} \vee \mu_{B}, \gamma_{A} \wedge \gamma_{B}\right)$,

(5) $\square A=\left(\mu_{A}, \bar{\mu}_{A}\right)$ where $\bar{\mu}_{A}=1-\mu_{A}$,

(6) $\nabla A=\left(\bar{\gamma}_{A}, \gamma_{A}\right)$ where $\bar{\gamma}_{A}=1-\gamma_{A}$.

The class of all intuitionistic fuzzy sets in $X$ will be denoted by $I F(X)$.

DEFINITION 2.5 Let $U$ be an initial universe and $E$ be the set of parameters. Let $A \subseteq E$. A pair $(\hat{F}, A)$ is called an intuitionistic fuzzy soft set over $U$, where $\hat{F}$ is a mapping given by $\hat{F}: A \rightarrow I F(U)$.

In general, for every $\delta \in A . \hat{F}[\delta]=\left\langle\mu_{\hat{F}[\delta]}, \gamma_{\hat{F}[\delta]}\right\rangle$ is an intuitionistic fuzzy set in $U$ and it is called intuitionistic fuzzy value set of parameter $\delta$.

DEFINITION 2.6 Let $U$ be an initial universe and $E$ be a set of parameters. Suppose that $A, B \subseteq E,(\hat{F}, A)$ and $(\hat{G}, B)$ be two intuitionistic fuzzy soft sets, we say that $(\hat{F}, A)$ is an intuitionistic fuzzy soft subset of $(\hat{G}, B)$ if and only

(1) $A \subseteq B$,

(2) for all $\delta \in A, \hat{F}[\delta]$ is an intuitionistic fuzzy subset of $\hat{G}[\delta]$, that is, for all $x \in U$ and $\delta \in A, \mu_{\hat{F}[\delta]}(x) \leq \mu_{\hat{G}[\delta]}(x)$, and $\gamma_{\hat{\hat{F}}[\delta]}(x) \geq \gamma_{\hat{G}[\delta]}(x)$. This relationship is denoted by $(\hat{F}, A) \hat{\subseteq}(\hat{G}, B)$.
Definition 2.7 Let $(\hat{F}, A)$ and $(\hat{G}, B)$ be two intuitionistic fuzzy soft sets over a common universe $U$. Then $(\hat{F}, A) \hat{\wedge}$ $(\hat{G}, B)$ is defined by $(\hat{F}, A) \hat{\wedge}(\hat{G}, B)=(\hat{\Xi}, A \times B)$, where $\hat{\Xi}[\delta, \varepsilon]=\hat{F}[\delta] \cap \hat{G}[\varepsilon]$ for all $(\delta, \varepsilon) \in A \times B$, that is,

$$
\hat{\Xi}[\delta, \varepsilon]=\left\langle\mu_{\hat{F}[\delta]}(x) \wedge \mu_{\hat{G}[\varepsilon]}(x), \gamma_{\hat{F}[\delta]}(x) \vee \gamma_{\hat{G}[\varepsilon]}(x)\right\rangle,
$$

for all $(\delta, \varepsilon) \in A \times B$, and $x \in U$.

Definition 2.8 Let $(\hat{F}, A)$ and $(\hat{G}, B)$ be two intuitionistic fuzzy soft sets over a common universe $U$. Then $(\hat{F}, A) \hat{\vee}$ $(\hat{G}, B)$ is defined by $(\hat{F}, A) \hat{\vee}(\hat{G}, B)=(\hat{\Omega}, A \times B)$, where $\hat{\Omega}[\delta, \varepsilon]=\hat{F}[\delta] \cup \hat{G}[\varepsilon]$ for all $(\delta, \varepsilon) \in A \times B$, that is,

$$
\hat{\Omega}[\delta, \varepsilon]=\left\langle\mu_{\hat{F}[\delta]}(x) \vee \mu_{\hat{G}[\varepsilon]}(x), \gamma_{\hat{F}[\delta]}(x) \wedge \gamma_{\hat{G}[\varepsilon]}(x)\right\rangle,
$$

for all $(\delta, \varepsilon) \in A \times B$, and $x \in U$.

DeFinition 2.9 Let $(\hat{F}, A)$ and $(\hat{G}, B)$ be two intuitionistic fuzzy soft sets over a common universe $U$. Then the intersection $(\hat{\Xi}, C)$, where $C=A \cup B$ and for all $\delta \in C$ and $x \in U$,

$$
\begin{aligned}
& \mu_{\hat{\Xi}[\delta]}(x)= \begin{cases}\mu_{\hat{\mathrm{F}}[\delta]}(x) & \text { if } \delta \in A-B \\
\mu_{\hat{G}[\delta]}(x) & \text { if } \delta \in B-A \\
\mu_{\hat{\mathrm{F}}[\delta]}(x) \wedge \mu_{\hat{\mathrm{F}}[\delta]}(x) & \text { if } \delta \in A \cap B,\end{cases} \\
& \gamma_{\hat{\hat{\mathrm{I}}}[\delta]}(x)= \begin{cases}\gamma_{\hat{\mathrm{F}}[\delta]}(x) & \text { if } \delta \in A-B \\
\gamma_{\hat{\mathrm{G}}[\delta]}(x) & \text { if } \delta \in B-A \\
\gamma_{\hat{\mathrm{F}}[\delta]}(x) \vee \gamma_{\hat{\mathrm{G}}[\delta]}(x) & \text { if } \delta \in A \cap B .\end{cases}
\end{aligned}
$$

We denote it by $(\hat{F}, A) \cap(\hat{G}, B)=(\hat{\Xi}, C)$.

DeFinition 2.10 Let $(\hat{F}, A)$ and $(\hat{G}, B)$ be two intuitionistic fuzzy soft sets over a common universe $U$. Then the union $(\breve{\Xi}, C)$, where $C=A \cup B$ and for all $\delta \in C$ and $x \in U$,

$$
\begin{aligned}
& \mu_{\hat{\Xi}[\delta]}(x)= \begin{cases}\mu_{\hat{F}[\delta]}(x) & \text { if } \delta \in A-B \\
\mu_{\hat{G}[\delta]}(x) & \text { if } \delta \in B-A \\
\mu_{\hat{F}[\delta]}(x) \vee \mu_{\hat{F}[\delta]}(x) & \text { if } \delta \in A \cap B,\end{cases} \\
& \gamma_{\hat{\hat{E}}[\delta]}(x)= \begin{cases}\gamma_{\hat{F}[\delta]}(x) & \text { if } \delta \in A-B \\
\gamma_{\hat{G}[\delta]}(x) & \text { if } \delta \in B-A \\
\gamma_{\hat{F}[\delta]}(x) \wedge \gamma_{\hat{G}[\delta]}(x) & \text { if } \delta \in A \cap B .\end{cases}
\end{aligned}
$$

We denote it by $(\hat{F}, A) \cup(\hat{G}, B)=(\hat{\Xi}, C)$.

In contrast with the above definitions of IF-soft set union and intersection, we may sometimes adopt different definitions of union and intersection given as follows. 
DeFinition 2.11 Let $(\hat{F}, A)$ and $(\hat{G}, B)$ be two IF-soft sets over a common universe $U$ and $A \cap B \neq \phi$. Then the bi-intersection of $(\hat{F}, A)$ and $(\hat{G}, B)$ is defined to be the fuzzy soft set $(\hat{\Xi}, C)$, where $C=A \cap B$ and $\hat{\Xi}[\delta]=\hat{F}[\delta] \cap \hat{G}[\delta]$, for all $\delta \in C$. This is denoted by $(\hat{\Xi}, C)=(\hat{F}, A) \hat{\cap}(\hat{G}, B)$.

DeFinition 2.12 Let $(\hat{F}, A)$ and $(\hat{G}, B)$ be two IF-soft sets over a common universe $U$ and $A \cap B \neq \phi$. Then the bi-union of $(\hat{F}, A)$ and $(\hat{G}, B)$ is defined to be the fuzzy soft set $(\hat{\Xi}, C)$, where $C=A \cap B$ and $\hat{\Xi}[\delta]=\hat{F}[\delta] \cup \hat{G}[\delta]$, for all $\delta \in C$. This is denoted by $(\hat{\Xi}, C)=(\hat{F}, A) \hat{\cup}(\hat{G}, B)$.

Definition 2.13 Let $A=\left(\mu_{A}, \gamma_{A}\right)$ be an intuitionistic fuzzy set. For arbitrary $\lambda, \theta \in[0,1]$, let the set

$$
A_{(\lambda, \theta)}=\left\{x \in X: \mu_{A}(x) \geq \lambda, \gamma_{A}(x) \leq \theta\right\},
$$

then $A_{(\lambda, \theta)}$ is called $a(\lambda, \theta)$-level set of $A$.

DEFINITION 2.14 [23] By a t-norm $\Delta$, we mean a function $\Delta:[0,1] \times[0,1] \rightarrow[0,1]$ satisfying the following conditions

(1) $x \Delta 1=x$

(2) $x \Delta y=y \Delta x$

(3) $x \Delta(y \Delta z)=(x \Delta y) \Delta z$

(4) if $w \leq x$ and $y \leq z$ then $w \Delta y \leq x \Delta z$

for all $x, y, z, w \in[0,1]$.

DEFINITION 2.15 [23] By an s-norm $\nabla$, we mean a function $\nabla:[0,1] \times[0,1] \rightarrow[0,1]$ satisfying the following conditions

(1) $x \nabla 0=x$

(2) $x \nabla y=y \nabla x$

(3) $x \nabla(y \nabla z)=(x \nabla y) \nabla z$

(4) if $w \leq x$ and $y \leq z$ then $w \nabla y \leq x \nabla z$

for all $x, y, z, w \in[0,1]$.

Definition 2.16 [23] A mapping $\eta:[0,1] \rightarrow[0,1]$ is called a negation if it satisfies

(1) $\eta(0)=1, \eta(1)=0$.

(2) $\eta$ is non-increasing.

(3) $\eta(\eta(x))=x$.

for all $x \in[0,1]$.

The most frequently used negation is $x \rightarrow 1-x$.

REMARK 2.17 [23] The t-norm and s-norm are said to be dual with respect to the negation $\eta(x)=1-x$, if

$$
x \nabla y=\eta(\eta(x) \Delta \eta(y)) .
$$

This holds with respect to $\eta$ if and only if $x \Delta y=\eta(\eta(x) \nabla \eta(y))$.
If for all $x \in[0,1], x \Delta x=x$ and $x \nabla x=x$, then $\Delta$ is called an idempotent t-norm and $\nabla$ is called an idempotent $\mathrm{t}$-conorm. There are many examples of $\mathrm{t}$-norms. Among the most known, we recall the following:

1. minimum: $x \Delta_{M} y=\min (x, y)$;

2. product: $x \Delta_{P} y=x \cdot y$;

3. Lukasiewics t-norm: $x \Delta y=\max \{x+y-1,0\}$;

4. drastic product: $x \Delta_{D} y=0$, if $(x, y) \in[0,1)^{2}$ and $x \Delta_{D} y=\min (x, y)$ otherwise.

\section{Intuitionistic Fuzzy Soft Groups}

In this section, we introduce the concept of intuitionistic fuzzy soft groups and investigate some related properties. Throughout the paper $G$ will denote a classical group.

Definition 3.1 Let $(\hat{F}, A)$ be an intuitionistic fuzzy soft set over a group $G$. Then $(\hat{F}, A)=\{\hat{F}[\delta]: \delta \in A\}$ is said to be an intuitionistic fuzzy soft group over $G$ if for all $\delta \in A, \hat{F}[\delta]=$ $\left\langle\mu_{\hat{F}[\delta]]}, \gamma_{\hat{F}[\delta]}\right\rangle$ is an intuitionistic fuzzy subgroup of $G$, where $\hat{F}[\delta]$ is a mapping given by $\hat{F}[\delta]: G \rightarrow[0,1]$.

DeFinition 3.2 Let $G$ be a classical group and $(\hat{F}, A)$ be an intuitionistic fuzzy soft set over $G$. Then $(\hat{F}, A)$ is called an intuitionistic fuzzy soft group over $G$ if it satisfies

(1) $\mu_{\hat{F}[\delta]}(x \cdot y) \geq \mu_{\hat{F}[\delta]}(x) \Delta \mu_{\hat{\hat{F}}[\delta]}(y)$ and $\gamma_{\hat{F}[\delta]}(x \cdot y) \leq \gamma_{\hat{F}[\delta]}(x) \nabla$ $\gamma_{\hat{F}[\delta]}(y)$,

(2) $\mu_{\hat{F}[\delta]}(x)=\mu_{\hat{F}[\delta]}\left(x^{-1}\right)$ and $\gamma_{\hat{F}[\delta]}(x)=\gamma_{\hat{[}[\delta]}\left(x^{-1}\right)$,

for all $x, y \in G$ and $\delta \in A$.

ExAmple 3.3 Let $G=\{e, x, y, z\}$ be the group with the binary operation defined below:

\begin{tabular}{|l|l|l|l|l|}
\hline$\cdot$ & $e$ & $x$ & $y$ & $z$ \\
\hline$e$ & $e$ & $x$ & $y$ & $z$ \\
\hline$x$ & $x$ & $z$ & $e$ & $y$ \\
\hline$y$ & $y$ & $e$ & $z$ & $x$ \\
\hline$z$ & $z$ & $y$ & $x$ & $e$ \\
\hline
\end{tabular}

Let $A=\{\delta, \varepsilon, \kappa, \xi\}$ be the set of parameters. For each parameter $\delta \in A, \hat{F}[\delta]$ is an intuitionistic fuzzy subgroup of $\mathrm{G}$, where $\hat{F}[\delta]$ is a mapping given by $\hat{F}[\delta]: G \rightarrow[0,1]$. For each parameter we define

$$
\begin{aligned}
\hat{F}[\delta]= & \{\langle e, 0.65,0.34\rangle,\langle x, 0.75,0.25\rangle,\langle y, 0.71,0.22\rangle, \\
& \langle z, 0.67,0.32\rangle\},
\end{aligned}
$$




$$
\begin{aligned}
\hat{F}[\varepsilon]= & \{\langle e, 0.88,0.12\rangle,\langle x, 0.83,0.11\rangle,\langle y, 0.71,0.19\rangle, \\
& \langle z, 0.75,0.21\rangle\}, \\
\hat{F}[\kappa]= & \{\langle e, 0,72,0.21\rangle,\langle x, 0.69,0.31\rangle,\langle y, 0.84,0.16\rangle, \\
& \langle z, 0.79,0.19\rangle\}, \\
\hat{F}[\xi]= & \{\langle e, 0.69,0.31\rangle,\langle x, 0.58,0.41\rangle,\langle y, 0.62,0.32\rangle, \\
& \langle z, 0.71,0.27\rangle\} .
\end{aligned}
$$

Corresponding t-norm and s-norm are defined as

$$
a \Delta b=\max \{a+b-1,0\} \text { and } a \nabla b=\min \{a+b, 1\} \text {, }
$$

for all $a, b \in[0,1]$. Each $\hat{F}[\delta]$ satisfies the conditions of intuitionistic fuzzy subgroup of $G$. Hence $(\hat{F}, A)=\{\hat{F}[\delta]: \delta \in A\}$ is an IF-soft group over $G$.

THEOREM 3.4 An IF-softset $(\hat{F}, A)$ over $G$ is an IF-softgroup over $G$ if and only iffor all $\delta \in A, \mu_{\hat{F}[\delta]}\left(x \cdot y^{-1}\right) \geq \mu_{\hat{F}[\delta]}(x) \Delta \mu_{\hat{F}[\delta]}(y)$ and $\gamma_{\hat{F}[\delta]}\left(x \cdot y^{-1}\right) \leq \gamma_{\hat{F}[\delta]}(x) \nabla \gamma_{\hat{F}[\delta]}(y)$ for every $x, y \in G$.

Proof. Suppose that $(\hat{F}, A)$ be an IF-soft group over $G$. By definition we have

$$
\mu_{\hat{F}[\delta]}\left(x \cdot y^{-1}\right) \geq \mu_{\hat{F}[\delta]}(x) \Delta \mu_{\hat{F}[\delta]}\left(y^{-1}\right)=\mu_{\hat{F}[\delta]}(x) \Delta \mu_{\hat{F}[\delta]}(y),
$$

and

$$
\gamma_{\hat{F}[\delta]}\left(x \cdot y^{-1}\right) \leq \gamma_{\hat{F}[\delta]}(x) \nabla \gamma_{\hat{F}[\delta]}\left(y^{-1}\right)=\gamma_{\hat{F}[\delta]}(x) \nabla \gamma_{\hat{F}[\delta]}(y) .
$$

This implies that $\mu_{\hat{F}[\delta]}\left(x \cdot y^{-1}\right) \geq \mu_{\hat{F}[\delta]}(x) \Delta \mu_{\hat{F}[\delta]}(y)$ and $\gamma_{\hat{\hat{F}}[\delta]}\left(x \cdot y^{-1}\right) \leq \gamma_{\hat{F}[\delta]}(x) \nabla \gamma_{\hat{F}[\delta]}(y)$ for every $x, y \in G$ and $\delta \in A$.

Conversely, let $\mu_{\hat{\mathrm{F}}[\delta]}\left(x \cdot y^{-1}\right) \geq \mu_{\hat{\mathrm{F}}[\delta]}(x) \Delta \mu_{\hat{\mathrm{F}}[\delta]}(y)$ and $\gamma_{\hat{F}[\delta]}\left(x \cdot y^{-1}\right) \leq \gamma_{\hat{F}[\delta]}(x) \nabla \gamma_{\hat{F}[\delta]}(y)$ for every $x, y \in G$ and $\delta \in A$. We show that for each $\delta \in A, \hat{F}[\delta]=\left\langle\mu_{\hat{F}[\delta]}, \gamma_{\hat{F}[\delta]}\right\rangle$ is an IF-subgroup of $G$. Since

$$
\mu_{\hat{F}[\delta]}(e \cdot x) \geq \mu_{\hat{F}[\delta]}(e) \Delta \mu_{\hat{F}[\delta]}(x)=\mu_{\hat{F}[\delta]}\left(x^{-1}\right),
$$

and

$$
\gamma_{\hat{F}[\delta]}(e \cdot x) \leq \gamma_{\hat{F}[\delta]}(e) \nabla \gamma_{\hat{F}[\delta]}(x)=\gamma_{\hat{F}[\delta]}\left(x^{-1}\right),
$$

for all $x \in G$ by supposition. This implies that $\mu_{\hat{F}[\delta]}(x) \geq \mu_{\hat{F}[\delta]}\left(x^{-1}\right)$ and $\gamma_{\hat{F}[\delta]}(x) \leq \gamma_{\hat{F}[\delta]}\left(x^{-1}\right)$ but then

$$
\mu_{\hat{F}[\delta]}\left(e \cdot x^{-1}\right) \geq \mu_{\hat{F}[\delta]}(e) \Delta \mu_{\hat{F}[\delta]}(x)=\mu_{\hat{F}[\delta]}(x),
$$

and

$$
\gamma_{\hat{F}[\delta]}\left(e \cdot x^{-1}\right) \leq \gamma_{\hat{F}[\delta]}(e) \nabla \gamma_{\hat{F}[\delta]}(x)=\gamma_{\hat{F}[\delta]}(x) .
$$

This implies that $\mu_{\hat{F}[\delta]}\left(x^{-1}\right) \geq \mu_{\hat{F}[\delta]}(x) \quad$ and $\gamma_{\hat{F}[\delta]}\left(x^{-1}\right) \leq \gamma_{\hat{F}[\delta]}(x)$. Thus we have $\mu_{\hat{F}[\delta]}\left(x^{-1}\right)=\mu_{\hat{F}[\delta]}(x)$ and $\gamma_{\hat{F}[\delta]}\left(x^{-1}\right)=\gamma_{\hat{F}[\delta]}(x)$ for all $x \in G$ and $\delta \in A$. Finally we show that $\mu_{\hat{F}[\delta]}(x \cdot y) \geq \mu_{\hat{F}[\delta]}(x) \Delta \mu_{\hat{F}[\delta]}(y)$ and $\gamma_{\hat{F}[\delta]}(x \cdot y) \leq \gamma_{\hat{F}[\delta]}(x) \nabla \gamma_{\hat{F}[\delta]}(y)$. Since

$$
\mu_{\hat{F}[\delta]}\left(x \cdot y^{-1}\right) \geq \mu_{\hat{F}[\delta]}(x) \Delta \mu_{\hat{F}[\delta]}\left(y^{-1}\right)=\mu_{\hat{F}[\delta]}(x) \Delta \mu_{\hat{F}[\delta]}(y),
$$

and

$$
\gamma_{\hat{\mathrm{F}}[\delta]}\left(x \cdot y^{-1}\right) \leq \gamma_{\hat{\mathrm{F}}[\delta]}(x) \nabla \gamma_{\hat{\mathrm{F}}[\delta]}\left(y^{-1}\right)=\gamma_{\hat{\mathrm{F}}[\delta]}(x) \nabla \gamma_{\hat{\mathrm{F}}[\delta]}(y) .
$$

This implies that $\mu_{\hat{F}[\delta]}(x \cdot y) \geq \mu_{\hat{F}[\delta]}(x) \Delta \mu_{\hat{F}[\delta]}(y)$ and $\gamma_{\hat{F}[\delta]}(x \cdot y) \leq \gamma_{\hat{F}[\delta]}(x) \nabla \gamma_{\hat{F}[\delta]}(y)$ for all $x, y \in G$. This completes the proof.

Theorem 3.5 Let $(\hat{F}, A)$ be an IF-soft group over $G$ and $x \in G$. Then for all $\delta \in A, \mu_{\hat{F}[\delta]}(x \cdot y)=\mu_{\hat{F}[\delta]}(y)$ and $\gamma_{\hat{F}[\delta]}(x \cdot y)=\gamma_{\hat{F}[\delta]}(y)$ for every $y \in G$ if $\mu_{\hat{F}[\delta]}(x)=\mu_{\hat{[}[\delta]}(e)$ and $\gamma_{\hat{\mathrm{F}}[\delta]}(x)=\gamma_{\hat{\mathrm{F}}[\delta]}(e)$.

TheOREM 3.6 Let $(\hat{F}, A)$ and $(\hat{G}, B)$ be two IF-soft groups over $G$. Then so are $(\hat{F}, A) \hat{\wedge}(\hat{G}, B)$ and $(\hat{F}, A) \hat{\cap}(\hat{G}, B)$.

Proof. We know that $(\hat{F}, A) \hat{\wedge}(\hat{G}, B)=(\hat{\Xi}, A \times B)$, where $\hat{\Xi}[\delta, \varepsilon]=\hat{F}[\delta] \cap \hat{G}[\varepsilon]$ for all $(\delta, \varepsilon) \in A \times B$, that is,

$$
\hat{\Xi}[\delta, \varepsilon]=\left\langle\mu_{\hat{F}[\delta]}(x) \wedge \mu_{\hat{F}[\delta]}(x), \gamma_{\hat{F}[\delta]}(x) \vee \gamma_{\hat{F}[\delta]}(x)\right\rangle,
$$

for all $(\delta, \varepsilon) \in A \times B$. Now for any $(\delta, \varepsilon) \in A \times B$, since $(\hat{F}, A)$ and $(\hat{G}, B)$ are IF-soft groups over $G$, for all $x, y \in G$ and $(\delta, \varepsilon) \in A \times B$, we have

$$
\begin{aligned}
\left(\mu_{\hat{\mathrm{F}}[\delta]} \wedge \mu_{\hat{\mathrm{G}}[\varepsilon]}\right)(x \cdot y) & =\mu_{\hat{\mathrm{F}}[\delta]}(x \cdot y) \wedge \mu_{\hat{\mathrm{G}}[\varepsilon]}(x \cdot y) \\
& \geq\left\{\mu_{\hat{\mathrm{F}}[\delta]}(x) \Delta \mu_{\hat{\mathrm{F}}[\delta]}(y)\right\} \wedge\left\{\mu_{\hat{\mathrm{G}}[\varepsilon]}(x) \Delta \mu_{\hat{\mathrm{G}}[\varepsilon]}(y)\right\} \\
& =\left\{\mu_{\hat{\mathrm{F}}[\delta]}(x) \wedge \mu_{\hat{\mathrm{G}}[\varepsilon]}(x)\right\} \Delta\left\{\mu_{\hat{\mathrm{F}}[\delta]}(y) \wedge \mu_{\hat{\mathrm{G}}[\varepsilon]}(y)\right\} \\
& =\left(\mu _ { \hat { \mathrm { F } } [ \delta ] } \wedge \mu _ { \hat { \mathrm { G } } [ \varepsilon ] } ( x ) \Delta \left(\mu_{\hat{\mathrm{F}}[\delta]} \wedge \mu_{\hat{\mathrm{G}}[\varepsilon]}(y),\right.\right.
\end{aligned}
$$

also

$$
\begin{aligned}
\left(\mu_{\hat{\mathrm{F}}[\delta]} \wedge \mu_{\hat{\mathrm{G}}[\varepsilon]}\right)\left(x^{-1}\right) & =\mu_{\hat{\mathrm{F}}[\delta]}\left(x^{-1}\right) \wedge \mu_{\hat{\mathrm{G}}[\delta]}\left(x^{-1}\right) \\
& =\mu_{\hat{\mathrm{F}}[\delta]}(x) \wedge \mu_{\hat{\mathrm{G}}[\varepsilon]}(x)=\left(\mu_{\hat{\mathrm{F}}[\delta]} \wedge \mu_{\hat{\mathrm{G}}[\delta]}\right)(x),
\end{aligned}
$$

and 


$$
\begin{aligned}
& \left(\gamma_{\hat{\mathrm{F}}[\delta]} \vee \gamma_{\hat{G}[\varepsilon]}\right)(x \cdot y)=\gamma_{\hat{\hat{F}}[\delta]}(x \cdot y) \vee \gamma_{\hat{G}[\varepsilon]}(x \cdot y) \\
& \leq\left\{\gamma_{\hat{[}[\delta]}(x) \nabla \gamma_{\hat{\hat{[}}[\delta]}(y)\right\} \vee\left\{\gamma_{\hat{G}[\varepsilon]}(x) \nabla \gamma_{\hat{G}[\varepsilon]}(y)\right\} \\
& =\left\{\gamma_{\hat{\mathrm{F}} \delta \delta]}(x) \vee \gamma_{\hat{\mathrm{G}}[\varepsilon]}(x)\right\} \nabla\left\{\gamma_{\hat{\mathrm{F}} \delta \delta]}(y) \vee \gamma_{\hat{\mathrm{G}}[\varepsilon]}(y)\right\} \\
& \left.=\left(\gamma_{\hat{\hat{H}} \delta]} \vee \gamma_{\hat{\mathrm{G}}[\varepsilon]}\right)(x) \nabla\left(\gamma_{\hat{\mathrm{H}}[\delta]} \vee \gamma_{\hat{\mathrm{G}}[\varepsilon]}\right)(y)\right\} \text {, }
\end{aligned}
$$

also

$$
\begin{aligned}
\left(\gamma_{\hat{F}[\delta]} \vee \gamma_{\hat{G}[\varepsilon]}\right)\left(x^{-1}\right) & =\gamma_{\hat{F}[\delta]}\left(x^{-1}\right) \vee \gamma_{\hat{G}[\varepsilon]}\left(x^{-1}\right) \\
& =\gamma_{\hat{F}[\delta]}(x) \vee \gamma_{\hat{G}[\varepsilon]}(x)=\left(\gamma_{\hat{F}[\delta]} \vee \gamma_{\hat{G}[\varepsilon]}\right)(x) .
\end{aligned}
$$

This shows that $(\hat{F}, A) \hat{\wedge}(\hat{G}, B)$ is an IF-soft group over G. The proof of $(\hat{F}, A) \hat{\cap}(\hat{G}, B)$ is similar to the proof of $(\hat{F}, A) \hat{\wedge}(\hat{G}, B)$.

Theorem 3.7 Let $(\hat{F}, A)$ and $(\hat{G}, B)$ be two IF-soft groups over $G$. Then so are $(\hat{F}, A) \hat{\vee}(\hat{G}, B)$ and $(\hat{F}, A) \hat{\cup}(\hat{G}, B)$.

Proof. We know that $(\hat{F}, A) \hat{\vee}(\hat{G}, B)=(\hat{\Xi}, A \times B)$, where $\hat{\Xi}[\delta, \varepsilon]=\hat{F}[\delta] \cup \hat{G}[\varepsilon]$ for all $(\delta, \varepsilon) \in A \times B$, that is,

$$
\hat{\Xi}[\delta, \varepsilon]=\left\langle\mu_{\hat{F}[\delta]}(x) \vee \mu_{\hat{G}[\varepsilon]}(x), \gamma_{\hat{F}[\delta]}(x) \wedge \gamma_{\hat{G}[\varepsilon]}(x)\right\rangle,
$$

for all $(\delta, \varepsilon) \in A \times B$. Now for any $(\delta, \varepsilon) \in A \times B$, since $(\hat{F}, A)$ and $(\hat{G}, B)$ are IF-soft groups over $G$, for all $x, y \in G$ and $(\delta, \varepsilon) \in A \times B$, we have

$$
\begin{aligned}
\left(\mu_{\hat{F}[\delta]} \vee \mu_{\hat{G}[\varepsilon]}\right)(x \cdot y) & =\mu_{\hat{F}[\delta]}(x \cdot y) \vee \mu_{\hat{G}[\varepsilon]}(x \cdot y) \\
& \geq\left\{\mu_{\hat{F}[\delta]}(x) \Delta \mu_{\hat{F}[\delta]}(y)\right\} \vee\left\{\mu_{\hat{G}[\varepsilon]}(x) \Delta \mu_{\hat{G}[\varepsilon]}(y)\right\} \\
& =\left\{\mu_{\hat{F}[\delta]}(x) \vee \mu_{\hat{G}[\varepsilon]}(x)\right\} \Delta\left\{\mu_{\hat{F}[\delta]}(y) \vee \mu_{\hat{G}[\varepsilon]}(y)\right\} \\
& =\left(\mu_{\hat{F}[\delta]} \vee \mu_{\hat{G}[\varepsilon]}\right)(x) \Delta\left(\mu_{\hat{F}[\delta]} \vee \mu_{\hat{G}[\varepsilon]}\right)(y),
\end{aligned}
$$

also

$$
\begin{aligned}
\left(\mu_{\hat{\mathrm{F}}[\delta]} \vee \mu_{\hat{G}[\varepsilon]}\right)\left(x^{-1}\right) & =\mu_{\hat{\mathrm{F}}[\delta]}\left(x^{-1}\right) \vee \mu_{\hat{\mathrm{G}}[\varepsilon]}\left(x^{-1}\right) \\
& =\mu_{\hat{\mathrm{F}}[\delta]}(x) \vee \mu_{\hat{\mathrm{G}}[\varepsilon]}(x)=\left(\mu_{\hat{\mathrm{F}}[\delta]} \vee \mu_{\hat{\mathrm{G}}[\varepsilon]}\right)(x),
\end{aligned}
$$

and

$$
\begin{aligned}
\left(\gamma_{\hat{\hat{F}}[\delta]} \wedge \gamma_{\hat{G}[\varepsilon]}\right)(x \cdot y) & =\gamma_{\hat{F}[\delta]}(x \cdot y) \wedge \gamma_{\hat{G}[\varepsilon]}(x \cdot y) \\
& \leq\left\{\gamma_{\hat{F}[\delta]}(x) \nabla \gamma_{\hat{F}[\delta]}(y)\right\} \wedge\left\{\gamma_{\hat{G}[\varepsilon]}(x) \nabla \gamma_{\hat{G}[\varepsilon]}(y)\right\} \\
& =\left\{\gamma_{\hat{F}[\delta]}(x) \wedge \gamma_{\hat{G}[\varepsilon]}(x)\right\} \nabla\left\{\gamma_{\hat{F}[\delta]}(y) \wedge \gamma_{\hat{G}[\varepsilon]}(y)\right\} \\
& =\left(\gamma_{\hat{\hat{F}}[\delta]} \vee \gamma_{\hat{G}[\varepsilon]}\right)(x) \nabla\left(\gamma_{\hat{\hat{F}}[\delta]} \vee \gamma_{\hat{G}[\varepsilon]}\right)(y),
\end{aligned}
$$

also

$$
\begin{aligned}
\left(\gamma_{\hat{\mathrm{F}}[\delta]} \wedge \gamma_{\hat{\mathrm{G}}[\varepsilon]}\right)\left(x^{-1}\right) & =\gamma_{\hat{\mathrm{F}}[\delta]}\left(x^{-1}\right) \wedge \gamma_{\hat{\mathrm{G}}[\varepsilon]}\left(x^{-1}\right) \\
& =\gamma_{\hat{\mathrm{F}}[\delta]}(x) \wedge \gamma_{\hat{\mathrm{G}}[\varepsilon]}(x)=\left(\gamma_{\hat{\mathrm{F}}[\delta]} \wedge \gamma_{\hat{\mathrm{G}}[\varepsilon]}\right)(x) .
\end{aligned}
$$

This shows that $(\hat{F}, A) \hat{\vee}(\hat{G}, B)$ is an IF-soft group over G. The proof of $(\hat{F}, A) \hat{\cup}(\hat{G}, B)$ is similar to the proof of $(\hat{F}, A) \hat{\vee}(\hat{G}, B)$.

TheOrem 3.8 Let $(\hat{F}, A)$ and $(\hat{G}, B)$ be two IF-soft groups over $G$. Then so is $(\hat{F}, A) \cap(\hat{G}, B)$.

Proof. We know that $(\hat{F}, A) \cap(\hat{G}, B)=(\hat{\Xi}, C)$, where $C=A \cup B$. Now for any $\delta \in C$ and $x, y \in G$, we consider the following cases

Case 1: For any $\delta \in A-B$, we have

$$
\begin{aligned}
\mu_{\hat{\Xi}[\delta]}(x \cdot y) & =\mu_{\hat{F}[\delta]}(x \cdot y) \geq \mu_{\hat{F}[\delta]}(x) \Delta \mu_{\hat{F}[\delta]}(y) \\
& =\mu_{\hat{\Xi}[\delta]}(x) \Delta \mu_{\hat{\Xi}[\delta]}(y),
\end{aligned}
$$

also $\mu_{\hat{\hat{E}}[\delta]}\left(x^{-1}\right)=\mu_{\hat{\hat{F}}[\delta]}\left(x^{-1}\right)=\mu_{\hat{F}[\delta]}(x)=\mu_{\hat{\Xi}[\delta]}(x)$, and

$$
\begin{aligned}
\gamma_{\hat{\hat{E}}[\delta]}(x \cdot y) & =\gamma_{\hat{\mathrm{F}}[\delta]}(x \cdot y) \leq \gamma_{\hat{\mathrm{F}}[\delta]}(x) \nabla \gamma_{\hat{\hat{F}}[\delta]}(y) \\
& =\gamma_{\hat{\hat{[}}[\delta]}(x) \nabla \gamma_{\hat{\hat{E}}[\delta]}(y),
\end{aligned}
$$

also $\gamma_{\hat{\hat{\Xi}}[\delta]}\left(x^{-1}\right)=\gamma_{\hat{\hat{F}}[\delta]}\left(x^{-1}\right)=\gamma_{\hat{\hat{F}}[\delta]}(x)=\gamma_{\hat{\hat{\Xi}}[\delta]}(x)$.

Case 2: For any $\delta \in B-A$, we have

$$
\begin{aligned}
\mu_{\hat{\Xi}[\delta]}(x \cdot y) & =\mu_{\hat{\hat{G}}[\delta]}(x \cdot y) \geq \mu_{\hat{\hat{G}}[\delta]}(x) \Delta \mu_{\hat{G}[\delta]}(y) \\
& =\mu_{\hat{\Xi}[\delta]}(x) \Delta \mu_{\hat{\hat{\Xi}}[\delta]}(y),
\end{aligned}
$$

also $\mu_{\hat{\hat{\Xi}}[\delta]}\left(x^{-1}\right)=\mu_{\hat{\mathrm{G}}[\delta]}\left(x^{-1}\right)=\mu_{\hat{\mathrm{G}}[\delta]}(x)=\mu_{\hat{\hat{\Xi}}[\delta]}(x)$, and

$$
\begin{aligned}
\gamma_{\hat{\Xi}[\delta]}(x \cdot y) & =\gamma_{\hat{G}[\delta]}(x \cdot y) \leq \gamma_{\hat{G}[\delta]}(x) \nabla \gamma_{\hat{G}[\delta]}(y) \\
& =\gamma_{\hat{\Xi}[\delta]}(x) \nabla \gamma_{\hat{\Xi}[\delta]}(y),
\end{aligned}
$$

also $\gamma_{\hat{\hat{E}}[\delta]}\left(x^{-1}\right)=\gamma_{\hat{G}[\delta]}\left(x^{-1}\right)=\gamma_{\hat{G}[\delta]}(x)=\gamma_{\hat{\Xi}[\delta]}(x)$.

Case 3: For any $\delta \in A \cap B$, we have $\mu_{\hat{\mathrm{F}}[\delta]} \cap \mu_{\hat{\mathrm{G}}[\varepsilon]}$ and $\gamma_{\hat{F}[\delta]} \cup \gamma_{\hat{G}[\varepsilon]}$. Analogous to the proof of Theorem 3.6, we have $\mu_{\hat{\Xi}[\delta]}(x \cdot y) \geq \mu_{\hat{\Xi}[\delta]}(x) \Delta \mu_{\hat{\Xi}[\delta]}(y)$ and $\gamma_{\hat{\Xi}[\delta]}(x \cdot y) \leq \gamma_{\hat{\Xi}[\delta]}(x) \nabla \gamma_{\hat{\Xi}[\delta]}(y)$,

also $\mu_{\hat{\hat{\Xi}}[\delta]}\left(x^{-1}\right)=\mu_{\hat{\hat{\Xi}}[\delta]}(x)$ and $\gamma_{\hat{\bar{\Xi}}[\delta]}\left(x^{-1}\right)=\gamma_{\hat{\bar{\Xi}}[\delta]}(x)$. Thus in any case we have $\mu_{\hat{\Xi}[\delta]}(x \cdot y) \geq \mu_{\hat{\hat{\Xi}}[\delta]}(x) \Delta \mu_{\hat{\hat{\Xi}}[\delta]}(y)$ and $\gamma_{\hat{\hat{\Xi}}[\delta]}(x \cdot y) \leq \gamma_{\hat{\hat{\Xi}}[\delta]}(x) \nabla \gamma_{\hat{\hat{\Xi}[\delta]}}(y)$. Thus $(\hat{F}, A) \cap(\hat{G}, B)$ is an IF-soft group over $G$.

TheOrem 3.9 Let $(\hat{F}, A)$ and $(\hat{G}, B)$ be two IF-soft groups over $G$. Then so is $(\hat{F}, A) \cup(\hat{G}, B)$.

DEFINITION 3.10 The necessity operation on an intuitionistic fuzzy soft set $(\hat{F}, A)$ is denoted by $\square(\hat{F}, A)$ and is defined as 


$$
\mathrm{D}(\hat{F}, A)=\left\{\left\langle x, \mu_{\mathrm{a} \hat{F}[\delta]}(x), \bar{\mu}_{\mathrm{a} \hat{F}[\delta]}(x)\right\rangle: x \in G \text { and } \delta \in A\right\} .
$$

Here $\mu_{\mathrm{a} \hat{\mathrm{F}}[\delta]}(x)$ is the fuzzy membership degree that object $x$ holds on parameter $\delta, \bar{\mu}_{\mathrm{a} \hat{\mathrm{F}} \delta \mid}$ is the fuzzy membership degree that object $x$ does not hold on parameter $\delta$, and $\hat{F}$ is a mapping $\hat{F}: A \rightarrow I F(G)$.

DeFINITION 3.11 The possibility operation on an intuitionistic fuzzy soft set $(\hat{F}, A)$ is denoted by $\diamond(\hat{F}, A)$ and is defined as

$$
\diamond(\hat{F}, A)=\left\{\left\langle x, \bar{\gamma}_{\Delta \hat{\mathrm{F}}[\delta]}(x), \gamma_{\Delta \hat{\mathrm{F}}[\delta]}(x)\right\rangle: x \in G \text { and } \delta \in A\right\} .
$$

Here $\gamma_{\diamond \hat{F}[\delta]}(x)$ is the fuzzy membership degree that object $x$ does not holds on parameter $\delta, \bar{\gamma}_{\diamond \hat{F}[\delta]}(x)$ is the fuzzy membership degree that object $x$ hold on parameter $\delta$, and $\hat{F}$ is a mapping $\hat{F}: A \rightarrow \operatorname{IF}(G)$.

TheOREM 3.12 Let $(\hat{F}, A)$ be an IF-soft group over a group G. Then

(1) $\mathrm{d}(\hat{F}, A)$ is an IF-soft group over a group $G$,

(2) $\diamond(\hat{F}, A)$ is an IF-soft group over a group $G$.

Proof. (1) Let $(\hat{F}, A)$ be an IF-soft group over a group $G$ and let for any $x, y \in G$ and $\delta \in A$, we have

$$
\begin{aligned}
\bar{\mu}_{\square \hat{\mathrm{F}}[\delta]}(x \cdot y) & =1-\mu_{\square \hat{\mathrm{F}}[\delta]}(x \cdot y) \\
& \leq 1-\left\{\mu_{\square \hat{[}[\delta]}(x) \Delta \mu_{\square \hat{\mathrm{F}}[\delta]}(y)\right\} \\
& =\eta\left\{\mu_{\square \hat{F}[\delta]}(x) \Delta \mu_{\square \hat{\mathrm{F}}[\delta]}(y)\right\} \\
& \left.=\eta\left(\mu_{\square \hat{\mathrm{F}}[\delta]}(x)\right) \nabla \eta \mu_{\square \hat{\mathrm{F}}[\delta]}(y)\right) \\
& =\left(1-\mu_{\square \hat{\mathrm{F}}[\delta]}(x)\right) \nabla\left(1-\mu_{\square \hat{\mathrm{F}}[\delta]}(y)\right) \\
& =\bar{\mu}_{\square \hat{\mathrm{F}}[\delta]}(x) \nabla \bar{\mu}_{\square \hat{\mathrm{F}}[\delta]}(y),
\end{aligned}
$$

also

$$
\bar{\mu}_{\square \hat{F}[\delta]}\left(x^{-1}\right)=1-\mu_{\square \hat{F}[\delta]}\left(x^{-1}\right)=1-\mu_{\square \hat{F}[\delta]}(x)=\bar{\mu}_{\square \hat{F}[\delta]}(x) .
$$

Hence $(\hat{F}, A)$ is an IF-soft group over $G$.

(2) The proof is similar to the proof of (1).

TheOREM 3.13 Let $G$ be a classical group and $(\hat{F}, A)$ be an IF-soft set over $G$. Then $(\hat{F}, A)$ is IF-soft group over $G$ iff for arbitrary $\lambda, \theta \in[0,1]$, if $\hat{F}[\delta]_{(\lambda, \theta)} \neq \phi$, then $(\hat{F}, A)_{(\lambda, \theta)}$ is a soft group over $G$.

Proof. Let $(\hat{F}, A)$ be an IF-soft group over $G$ and $x, y \in \hat{F}[\delta]_{(\lambda, \theta)}$, for arbitrary $\lambda, \theta \in[0,1]$ and $\delta \in A$. Then, we have $\mu_{\hat{\hat{F}}[\delta]}(x) \geq \lambda, \gamma_{\hat{F}[\delta]}(x) \leq \theta$ and $\mu_{\hat{F}[\delta]}(y) \geq \lambda, \gamma_{\hat{F}[\delta]}(y) \leq \theta$. Since $(\hat{F}, A)$ is an IF-soft group over $\mathrm{G}$, then we have

$$
\mu_{\hat{F}[\delta]}(x \cdot y) \geq \mu_{\hat{\hat{F}}[\delta]}(x) \Delta \mu_{\hat{\mathrm{F}}[\delta]}(y) \geq \lambda \Delta \lambda=\lambda,
$$

and

$$
\gamma_{\hat{\mathrm{F}}[\delta]}(x \cdot y) \leq \gamma_{\hat{\mathrm{F}}[\delta]}(x) \nabla \gamma_{\hat{\hat{F}}[\delta]}(y) \leq \theta \nabla \theta=\theta \text {. }
$$

Therefore, $\quad x \cdot y \in \hat{F}[\delta]_{(\lambda, \theta)}$. Furthermore $\mu_{\hat{F}[\delta]}\left(x^{-1}\right)=$ $\mu_{\hat{F}[\delta]}(x) \geq \lambda$ and $\gamma_{\hat{\hat{F}}[\delta]}\left(x^{-1}\right)=\gamma_{\hat{F}[\delta]}(x) \leq \theta$. So $x^{-1} \in \hat{F}[\delta]_{(\lambda, \theta)}$. Hence $(\hat{F}, A)_{(\lambda, \theta)}$ is a soft group over $G$. Conversely, suppose that,

$$
\begin{gathered}
\mu_{\hat{F}[\delta]}\left(x_{0} \cdot y_{0}^{-1}\right) \pm \mu_{\hat{F}[\delta]}\left(x_{0}\right) \Delta \mu_{\hat{F}[\delta]}\left(y_{0}\right) \text { and } \\
\gamma_{\hat{F}[\delta]}\left(x_{0} \cdot y_{0}^{-1}\right) \notin \gamma_{\hat{F}[\delta]}\left(x_{0}\right) \nabla \gamma_{\hat{F}[\delta]}\left(y_{0}\right),
\end{gathered}
$$

for $x_{0}, y_{0} \in G$ and $\delta \in A$. Let us assume that, $\mu_{\hat{F}[\delta]}\left(x_{0} \cdot y_{0}^{-1}\right) \geq$ $\mu_{\hat{F}[\delta]}\left(x_{0}\right) \Delta \mu_{\hat{F}[\delta]}\left(y_{0}\right)$. This implies

$$
\mu_{\hat{\mathrm{F}}[\delta]}\left(x_{0} \cdot y_{0}^{-1}\right)<\mu_{\hat{\mathrm{F}}[\delta]}\left(x_{0}\right) \Delta \mu_{\hat{\mathrm{F}}[\delta]}\left(y_{0}\right) .
$$

Let $\mu_{\hat{F}[\delta]}\left(x_{0}\right)=\lambda_{1}, \quad \mu_{\hat{F}[\delta]}\left(y_{0}\right)=\lambda_{2}$, and $\mu_{\hat{F}[\delta]}\left(x_{0} \cdot y_{0}^{-1}\right)=\lambda_{3}$. If we take $\lambda=\lambda_{1} \Delta \lambda_{2}$, then $x_{0} \cdot y_{0}^{-1} \notin \hat{F}[\delta]_{(\lambda, \theta)}$. For each $\theta$,

$$
\mu_{\hat{F}[\delta]}\left(x_{0}\right)=\lambda_{1} \geq \lambda_{1} \Delta \lambda_{2}=\lambda,
$$

and

$$
\mu_{\hat{F}[\delta]}\left(y_{0}\right)=\lambda_{2} \geq \lambda_{1} \Delta \lambda_{2}=\lambda
$$

But by choosing $\theta$, satisfying the condition $\gamma_{\hat{F}[\delta]}\left(x_{0}\right) \leq \theta$ and $\gamma_{\hat{F}[\delta]}\left(y_{0}\right) \leq \theta$, we have $x_{0}, y_{0} \in \hat{F}[\delta]_{(\lambda, \theta)}$. This contradict with the fact that $(\hat{F}, A)_{(\lambda, \theta)}$ is a soft group over $G$. In case $\gamma_{\hat{F}[\delta]}\left(x_{0} \cdot y_{0}^{-1}\right) \pm \gamma_{\hat{F}[\delta]}\left(x_{0}\right) \nabla \gamma_{\hat{F}[\delta]}\left(y_{0}\right)$, we can obtain this result in similar way.

THeOrem 3.14 Let $(\hat{F}, A)$ and $(\hat{G}, B)$ be two IF-soft groups over $G$. Then

(1) $((\hat{F}, A) \hat{\cap}(\hat{G}, B))_{(\lambda, \theta)}=(\hat{F}, A)_{(\lambda, \theta)} \hat{\cap}(\hat{G}, B)_{(\lambda, \theta)}$,

(2) $((\hat{F}, A) \hat{\cup}(\hat{G}, B))_{(\lambda, \theta)}=(\hat{F}, A)_{(\lambda, \theta)} \hat{\cup}(\hat{G}, B)_{(\lambda, \theta)}$,

(3) $((\hat{F}, A) \cap(\hat{G}, B))_{(\lambda, \theta)}=(\hat{F}, A)_{(\lambda, \theta)} \cap(\hat{G}, B)_{(\lambda, \theta)}$,

(4) $((\hat{F}, A) \cup(\hat{G}, B))_{(\lambda, \theta)}=(\hat{F}, A)_{(\lambda, \theta)} \cup(\hat{G}, B)_{(\lambda, \theta)}$.

Proof. (1) Let $(\hat{F}, A)$ and $(\hat{G}, B)$ be two IF-soft groups over $G$ and $A \cap B \neq \phi$. We know that $(\hat{\Xi}, C)=(\hat{F}, A) \hat{\cap}(\hat{G}, B)$, where $C=A \cap B$ and $\hat{\Xi}[\delta]=\hat{F}[\delta] \cap \hat{G}[\delta]$ for all $\delta \in C$. Now for any $\delta \in C$ and $x \in G$, we have 


$$
\begin{aligned}
& x \in\left(\mu_{\hat{F}[\delta]} \cap \mu_{\hat{G}[\delta]}\right)_{(\lambda, \theta)} \\
& \Leftrightarrow \mu_{\hat{F}[\delta]}(x) \wedge \mu_{\hat{G}[\delta]}(x) \geq \lambda \\
& \Leftrightarrow \mu_{\hat{\hat{F}}[\delta]}(x) \geq \lambda \text { and } \mu_{\hat{G}[\delta]}(x) \geq \lambda \\
& \Leftrightarrow x \in\left(\mu_{\hat{F}[\delta]}\right)_{(\lambda, \theta)} \cap\left(\mu_{\hat{G}[\delta]}\right)_{(\lambda, \theta)},
\end{aligned}
$$

and

$$
\begin{aligned}
& x \in\left(\gamma_{\hat{F}[\delta]} \cup \gamma_{\hat{G}[\delta]}\right)_{(\lambda, \theta)} \\
& \Leftrightarrow \gamma_{\hat{F}[\delta]}(x) \vee \gamma_{\hat{G}[\delta]}(x) \leq \theta \\
& \Leftrightarrow \gamma_{\hat{F}[\delta]}(x) \leq \theta \text { or } \gamma_{\hat{G}[\delta]}(x) \leq \theta \\
& \Leftrightarrow x \in\left(\gamma_{\hat{F}[\delta]}\right)_{(\lambda, \theta)} \cup\left(\gamma_{\hat{G}[\delta]}\right)_{(\lambda, \theta)} .
\end{aligned}
$$

This shows that $((\hat{F}, A) \hat{\cap}(\hat{G}, B))_{(\lambda, \theta)}=(\hat{F}, A)_{(\lambda, \theta)} \hat{\cap}(\hat{G}, B)_{(\lambda, \theta)}$.

The proofs of (2), (3) and (4) are similar to the proof of (1).

\section{Cartesian Product of Intuitionistic Fuzzy Soft Groups}

In this section, we define cartesian product of IF-soft groups and investigate some related properties.

Definition 4.1 Let $(\hat{F}, A)$ and $(\hat{G}, B)$ be two intuitionistic fuzzy soft sets over $G_{1}$ and $G_{2}$, respectively. Then the cartesian product of IF-soft sets $(\hat{F}, A)$ and $(\hat{G}, B)$ is defined as

$$
(\hat{F}, A) \otimes(\hat{G}, B)=(\hat{P}, A \times B),
$$

where $\hat{P}[\delta, \vartheta]=\hat{F}[\delta] \times \hat{G}[\vartheta]=\left\langle\mu_{\hat{F}[\delta] \times \hat{G}[\vartheta],} \gamma_{\hat{F}[\delta] \times \hat{G}[\vartheta]}\right\rangle$ for all $(\delta, \vartheta) \in A \times B$.

Here $\mu_{\hat{\mathrm{F}}[\delta] \times \hat{G}[\vartheta]}(x, y)=\mu_{\hat{\mathrm{F}}[\delta]}(x) \wedge \mu_{\hat{G}[\vartheta]}(y)$ and $\gamma_{\hat{\mathrm{F}}[\delta] \times \hat{G}[\vartheta:}$ $(x, y)=\gamma_{\hat{F}[\delta]}(x) \vee \gamma_{\hat{G}[\vartheta]}(y)$ for all $(x, y) \in G_{1} \times G_{2}$ and $(\delta, \vartheta)$ $\in A \times B$.

DefinITION 4.2 An intuitionistic fuzzy soft $\operatorname{set}(\hat{F}, A) \otimes(\hat{G}, B)$ over $G_{1} \times G_{2}$ is called an intuitionistic fuzzy soft group over $G_{1} \times G_{2}$ if it satisfies

$$
\begin{aligned}
& \text { (1) } \mu_{\hat{\mathrm{F}}[\delta] \times \hat{G}[\vartheta]}\left(\left(x_{1}, y_{1}\right) \cdot\left(x_{2}, y_{2}\right)\right) \geq \\
& \mu_{\hat{\mathrm{F}}[\delta] \times \hat{G}[\vartheta]}\left(x_{1}, y_{1}\right) \Delta \mu_{\hat{\mathrm{F}}[\delta] \times \hat{G}[\vartheta]}\left(x_{2}, y_{2}\right) \text {; } \\
& \text { (2) } \gamma_{\hat{\mathrm{F}}[\delta] \times \hat{\mathrm{G}}[\vartheta]}\left(\left(x_{1}, y_{1}\right) \cdot\left(x_{2}, y_{2}\right)\right) \leq \\
& \gamma_{\hat{F}[\delta] \times \hat{G}[\vartheta]}\left(x_{1}, y_{1}\right) \nabla \gamma_{\hat{F}[\delta] \times \hat{G}[\vartheta]}\left(x_{2}, y_{2}\right) \text {; } \\
& \text { (3) } \mu_{\hat{\mathrm{F}}[\delta] \times \hat{\mathrm{G}}[\vartheta]}\left(x_{1}, y_{1}\right)=\mu_{\hat{\mathrm{F}}[\delta] \times \hat{\mathrm{G}}[\vartheta]}\left(\left(x_{1}, y_{1}\right)^{-1}\right) \text { and } \gamma_{\hat{\mathrm{F}}[\delta] \times \hat{\mathrm{G}}[\vartheta} \\
& \left(x_{1}, y_{1}\right)=\gamma_{\hat{F}[\delta] \times \hat{G}[\vartheta]}\left(\left(x_{1}, y_{1}\right)^{-1}\right) \text {, }
\end{aligned}
$$

for all $\left(x_{1}, y_{1}\right),\left(x_{2}, y_{2}\right) \in G_{1} \times G_{2}$ and $(\delta, \vartheta) \in A \times B$.
Theorem 4.3 Let $(\hat{F}, A)$ and $(\hat{G}, B)$ be two intuitionistic fuzzy soft groups over $G_{1}$ and $G_{2}$, respectively. Then the cartesian product $(\hat{F}, A) \otimes(\hat{G}, B)$ is an intuitionistic fuzzy soft group over $G_{1} \times G_{2}$.

Proof. Let $(\hat{F}, A)$ and $(\hat{G}, B)$ be two intuitionistic fuzzy soft groups over $G_{1}$ and $G_{2}$, respectively. For any $\left(x_{1}, y_{1}\right),\left(x_{2}, y_{2}\right) \in G_{1} \times G_{2}$ and $(\delta, \vartheta) \in A \times B$. We have

$$
\begin{aligned}
\mu_{\hat{F}[\delta] \times \hat{G}[\vartheta]}\left(\left(x_{1}, y_{1}\right) \cdot\left(x_{2}, y_{2}\right)\right)= & \mu_{\hat{F}[\delta] \times \hat{G}[\vartheta]}\left(x_{1} \cdot x_{2}, y_{1} \cdot y_{2}\right) \\
= & \mu_{\hat{F}[\delta]}\left(x_{1} \cdot x_{2}\right) \wedge \mu_{\hat{G}[\vartheta]}\left(y_{1} \cdot y_{2}\right) \\
\geq & \left\{\mu_{\hat{F}[\delta]}\left(x_{1}\right) \Delta \mu_{\hat{F}[\delta]}\left(x_{2}\right)\right\} \wedge \\
& \left\{\mu_{\hat{G}[\vartheta]}\left(y_{1}\right) \Delta \mu_{\hat{G}[\vartheta]}\left(y_{2}\right)\right\} \\
= & \left\{\mu_{\hat{F}[\delta]}\left(x_{1}\right) \wedge \mu_{\hat{G}[\vartheta]}\left(y_{1}\right)\right\} \Delta \\
& \left\{\mu_{\hat{F}[\delta]}\left(x_{2}\right) \wedge \mu_{\hat{G}[\vartheta]}\left(y_{2}\right)\right\} \\
= & \mu_{\hat{F}[\delta] \times \hat{G}[\vartheta]}\left(x_{1}, y_{1}\right) \Delta \\
& \mu_{\hat{F}[\delta] \times \hat{G}[\vartheta]}\left(x_{2}, y_{2}\right),
\end{aligned}
$$

also

$$
\begin{aligned}
\gamma_{\hat{F}[\delta] \times \hat{G}[\vartheta]}\left(\left(x_{1}, y_{1}\right) \cdot\left(x_{2}, y_{2}\right)\right)= & \gamma_{\hat{F}[\delta] \times \hat{G}[\vartheta]}\left(x_{1} \cdot x_{2}, y_{1} \cdot y_{2}\right) \\
= & \gamma_{\hat{F}[\delta]}\left(x_{1} \cdot x_{2}\right) \vee \gamma_{\hat{G}[\vartheta]}\left(y_{1} \cdot y_{2}\right) \\
\leq & \left\{\gamma_{\hat{F}[\delta]}\left(x_{1}\right) \nabla \gamma_{\hat{F}[\delta]}\left(x_{2}\right)\right\} \vee \\
& \left\{\gamma_{\hat{G}[\vartheta]}\left(y_{1}\right) \nabla \gamma_{\hat{G}[\vartheta]}\left(y_{2}\right)\right\} \\
= & \left\{\gamma_{\hat{F}[\delta]}\left(x_{1}\right) \vee \gamma_{\hat{G}[\vartheta]}\left(y_{1}\right)\right\} \nabla \\
& \left\{\gamma_{\hat{\hat{F}}[\delta]}\left(x_{2}\right) \vee \gamma_{\hat{G}[\vartheta]}\left(y_{2}\right)\right\} \\
= & \gamma_{\hat{F}[\delta] \times \hat{G}[\vartheta]}\left(x_{1}, y_{1}\right) \nabla \\
& \gamma_{\hat{F}[\delta] \times \hat{G}[\vartheta]}\left(x_{2}, y_{2}\right),
\end{aligned}
$$

and

$$
\begin{aligned}
\mu_{\hat{F}[\delta] \times \hat{G}[\vartheta]}\left(\left(x_{1}, y_{1}\right)^{-1}\right) & =\mu_{\hat{F}[\delta]}\left(x_{1}^{-1}\right) \wedge \mu_{\hat{G}[\vartheta]}\left(y_{1}^{-1}\right) \\
& =\mu_{\hat{F}[\delta]}\left(x_{1}\right) \wedge \mu_{\hat{G}[\vartheta]}\left(y_{1}\right) \\
& =\mu_{\hat{F}[\delta] \times \hat{G}[\vartheta]}\left(x_{1}, y_{1}\right),
\end{aligned}
$$

also

$$
\begin{aligned}
\gamma_{\hat{F}[\delta] \times \hat{G}[\vartheta]}\left(\left(x_{1}, y_{1}\right)^{-1}\right) & =\gamma_{\hat{F}[\delta]}\left(x_{1}^{-1}\right) \vee \gamma_{\hat{G}[\vartheta]}\left(y_{1}^{-1}\right) \\
& =\gamma_{\hat{F}[\delta]}\left(x_{1}\right) \vee \gamma_{\hat{G}[\vartheta]}\left(y_{1}\right) \\
& =\gamma_{\hat{F}[\delta] \times \hat{G}[\vartheta]}\left(x_{1}, y_{1}\right) .
\end{aligned}
$$

Hence this shows that $(\hat{F}, A) \otimes(\hat{G}, B)$ is an intuitionistic fuzzy soft group over $G_{1} \times G_{2}$. 


\section{Conclusion}

In this paper, we introduced the notion of the intuitionistic fuzzy soft groups and we studied this structure. In addition, we provided relationship between intuitionistic fuzzy soft groups and $(\lambda, \theta)$-level subsets. Finally, we proved some results on direct product of intuitionistic fuzzy soft groups under soft functions.

\section{Acknowledgements}

The authors are highly grateful to referees for their valuable comments and suggestions which were helpful in improving this paper.

\section{References}

1. Abdullah S, Davvaz B et al. (2011). $(\alpha, \beta)$-intuitionistic fuzzy ideals of hemirings, Computers \& Mathematics with Applications, vol 62(8), 3077-3090.

2. Abdullah S, Aslam M et al. (2012). Direct product of finite fuzzy subsets of LA-semigroups, Annals of Fuzzy Mathematics and Informatics, vol 3(2), 281-292.

3. Abdullah S, Satyanarayana B et al. (2012). Direct product of intuitionistic fuzzy $\mathrm{H}$-ideal in BCK-algebras, International Journal of Algebra and Statistics, vol 1(1), 8-16.

4. Abdullah S, Aslam M et al. (2012). A new type of fuzzy normal subgroups and fuzzy cosets, Journal of Intelligent and Fuzzy Systems, DOI 10.3233/IFS-2012-0612.

5. Akram M, and Dar KH (2007). Fuzzy left h-ideal in hemirings with respect to a s-norm, International Journal of Computational and Applied Mathematics, vol 1, 7-14.

6. Akram M (2007). Intuitionistic (S, T)-fuzzy Lie ideals of Lie algebras, Quasigroups Related Systems, vol 15, 201-218.

7. Aktas H, and Cagman N (2007). Soft sets and soft groups, Information Sciences, vol 177(13), 2726-2735.

8. Ali M I, and Shabir M (2009). Soft ideals and generalized fuzzy ideals in semigroups, New Mathematics and Natural Computation, vol 5(3), 599-615.

9. Ali M I, Feng F et al. (2009). On some new operations in soft set theory, Computers \& Mathematics with Applications, vol 57(9), 2621-2628.

10. Atanassov K T (1986). Intuitionistic fuzzy sets, Fuzzy Sets and Systems, vol 20(1), 87-96.

11. AtanassovKT(1999).Intuitionisticfuzzy sets, Physica-Verlag, Heidelberg, New York.

12. Aygunoglu A, Varol B P et al. (2012). Interval-valued intuitionistic fuzzy subgroups based on interval-valued double t-norm, Neural Computing and Applications, vol 21(1), 207-214.

13. Aygunoglu A, and Aygun H (2009). Introduction to fuzzy soft groups, Computers \& Mathematics with Applications, vol 58(6), 1279-1286.

14. Saeid A B, and Rezaei A (2012). Intuitionistic (T, S)-fuzzy CI-algebras, Computers \& Mathematics with Applications, vol 63, 158-166.

15. Feng F, Jun YB et al. (2008). Soft semirings, Computers \& Mathematics with Applications, vol 56(10), 2621-2628.

16. Hedayati $H(2012)$. Intuitionistic $(S, T)$-fuzzy $(1,2)$-ideals of semigroups with interval valued membership functions, International Journal of Fuzzy Systems vol 14(1), 154-159.

17. Jun Y B (2008). Soft BCK/BCI-algebras, Computers \& Mathematics with Applications, vol 56(5), 1408-1413.

18. Kim K H, and Lee J G (2008). Intuitionistic ( $T, S$ ) -normed fuzzy ideals of $\Gamma$-rings, International Mathematical Forum, vol 3(3), 115-123.

19. Maji P K, Biswas R et al. (2001). Intuitionistic fuzzy soft sets, Journal of Fuzzy Mathematics, vol 9(3), 677-692.

20. Maji P K, Roy A R et al. (2004). On intuitionistic fuzzy soft sets, Journal of Fuzzy Mathematics, vol 12(3), 669-683.

21. Maji PK, Biswas R and Roy AR (2001). Fuzzy soft sets, Journal of Fuzzy Mathematics, vol 9(3), 589-602.

22. Molodtsov D (1999). Soft set theory first results, Computers \& Mathematics with Applications, vol 37(4-5), 19-31.

23. Nguyen H T, and Walker E A (2006). A First Course in Fuzzy Logic, $3^{\text {rd }}$ Edn, Chapman and Hall/CRC Taylor and Francis Group.

24. Shum K P, and Akram M (2008). Intuitionistic (T, S) -fuzzy ideals of near-rings, Journal of Algebra Discrete Structures, vol 6(1), 37-52.

25. Yaqoob N, Aslam M et al (2012). On soft $\Gamma$-hyperideals over left almost $\Gamma$-semihypergroups, Journal of Advanced Research in Dynamical and Control Systems vol 4(1), 1-12.

26. Yaqoob N, Mostafa S M et al. (2013). On cubic KU-ideals of KU-algebras, ISRN Algebra, Article ID 935905, 10 pages.

27. Yaqoob N, Aslam M et al. (2012). Structures of N-Гhyperideals in left almost $\Gamma$-semihypergroups, World Applied Sciences Journal vol 17(12), 1611-1617.

28. Yaqoob N, Aslam M et al. (2012). Rough fuzzy hyperideals in ternary semihypergroups, Advances in Fuzzy Systems, Article ID 595687, 9 pages.

29. Zhan J (2005). On properties of fuzzy left h-ideals in hemirings with t-norms, International Journal of Mathematics and Mathematical Sciences, vol 2005(19), 3127-3144. 